\title{
Abnormalities of white matter integrity in the corpus callosum of adolescents with PTSD after childhood sexual abuse: a DTI study
}

\author{
Mirjam A. W. Rinne-Albers ${ }^{1}$ Steven J. A. van der Werff ${ }^{2,3} \cdot$ Marie-José van Hoof $^{1,3,4}$. \\ Natasja D. van Lang ${ }^{1}$. Francien Lamers-Winkelman ${ }^{5} \cdot$ Serge A. Rombouts $^{3,6,7}$. \\ Robert R. J. M. Vermeiren ${ }^{1,3,5} \cdot$ Nic J. A. van der $\mathrm{Wee}^{2,3}$
}

Received: 6 April 2015 / Accepted: 28 November 2015 / Published online: 23 December 2015

(C) The Author(s) 2015. This article is published with open access at Springerlink.com

\begin{abstract}
This study seeks to determine whether white matter integrity in the brain differs between adolescents with post-traumatic stress disorder (PTSD) due to childhood sexual abuse (CSA) and matched healthy adolescents and whether there is a relationship between white matter integrity and symptom severity in the patient group. Using 3T diffusion tensor imaging, we examined fractional anisotropy (FA) in a group of adolescents with CSA-related $\operatorname{PTSD}(n=20)$ and matched healthy controls $(n=20)$, in a region of interest consisting of the bilateral uncinate fasciculus (UF), the genu, splenium and body of the corpus callosum (CC), and the bilateral cingulum. In addition, we performed an exploratory whole brain analysis. Trauma symptomatology was measured with the Trauma Symptom Checklist for Children (TSCC) to enable correlational
\end{abstract}

Mirjam A.W. Rinne-Albers and Steven J.A. van der Werff share first authorship.

Mirjam A. W. Rinne-Albers

m.a.w.rinne@ curium.nl

1 Curium-LUMC, Academic Center for Child and Adolescent Psychiatry, Oegstgeest, The Netherlands

2 Department of Psychiatry, Leiden University Medical Center (LUMC), Leiden, The Netherlands

3 Leiden Institute for Brain and Cognition (LIBC), Leiden, The Netherlands

4 Psychotraumacenter and Department of Child and Adolescent Psychiatry, GGZ Rivierduinen, Leiden, The Netherlands

5 Institute of Psychology and Education, Vrije Universiteit, Amsterdam, The Netherlands

6 Department of Radiology, Leiden University Medical Center (LUMC), Leiden, The Netherlands

7 Institute of Psychology, Leiden University, Leiden, The Netherlands analyses between FA differences and trauma symptomatology. The PTSD group had significantly lower FA values in the genu, midbody and splenium of the $\mathrm{CC}$ in comparison with controls $(p<0.05$, tfce corrected). Post hoc analyses of the eigenvalues of the DTI scan showed increased radial and mean diffusivity in the patient group. In addition, we found a significant negative correlation between scores on the anger subscale of the TSCC and FA values in the left body of the CC in patients $(p<0.05)$. Adolescents with CSA-related PTSD show decreased FA in the CC, with abnormalities in the integrity of the left body of the CC being related to anger symptoms. These findings suggest that early trauma exposure affects the development of the $\mathrm{CC}$, which may play a role in the pathophysiology of PTSD in adolescents.

Keywords PTSD - Diffusion tensor imaging Adolescents $\cdot$ Sexual abuse $\cdot$ Neuroimaging

\section{Introduction}

Childhood psychotrauma is a prevalent and important predictor of both child and adult psychopathology as well as a number of somatic disorders [1, 26, 31, 45]. Preclinical research in rodents and non-human primates has shown that structure and functioning of the developing brain are highly vulnerable to the effects of adversity, especially in certain critical time windows. In animal studies, childhood adversity was found to be associated with changes in brain circuitry involved in stress and emotion regulation, such as the hippocampus and certain prefrontal regions, possibly underlying vulnerability to the impact of stressors later in life [12, 25, 32, 41]. In adult humans, a history of chronic traumatization during childhood and adolescence was 
found to be associated with structural and functional damage in key elements of emotion and stress regulating brain circuitry, for example in the hippocampus in adults reporting childhood abuse or in the medial prefrontal cortex in adults reporting childhood emotional maltreatment $[5,56]$. Bearing in mind the neuroplasticity of the maturing human brain [8], a thorough understanding of the human neurobiology underlying the psychological sequelae of childhood and youth psychotrauma may hold promise for developing appropriate interventions to alter adverse neurodevelopmental trajectories [22]. Some recent reviews and metaanalyses have addressed structural brain alterations following childhood trauma in both adolescents and (young) adults with and without psychopathology [11, 40, 60]. The studies included in these reviews and meta-analyses often used different approaches and varied in sample size. Nevertheless, next to findings in the cerebellum and sensory cortex, most of the results from reviews and meta-analyses point toward involvement of the corpus callosum (CC) and corticolimbic circuits in the pathophysiological sequelae of psychotrauma in children and young adults.

Diminished white matter (WM) integrity can constitute one element of abnormalities in corticolimbic and related circuitry. Diminished structural integrity may impede adaptive emotional and cognitive functioning, thereby rendering an individual vulnerable to childhood and adult psychopathology.

A promising tool for examining the structural integrity of WM in children and youth who experienced psychotrauma is diffusion tensor imaging (DTI). Fractional anisotropy (FA) is the most commonly used DTI parameter and reflects the degree of diffusion directionality of water, which in white matter can be influenced by structural properties such as axonal density, organization and myelinization. Smaller FA values are associated with decreased white matter integrity. To further interpret differences in FA, additional parameters such as the mean diffusivity (MD), axial diffusivity (AD), and radial diffusivity (RD) can be assessed as well.

So far, four studies in children and youth have employed DTI to examine the effects of psychotrauma on white matter integrity in the developing brain and several reported abnormalities in the $\mathrm{CC}$, but also in other areas. The first small study, in children who had been subjected to early socioemotional deprivation $(N=7)$, found decreased FA in the left UF [14]. The second study, in a group of children $(N=17)$ with post-traumatic stress disorder (PTSD) following varying forms of maltreatment, found reduced FA in the medial and posterior subregions of the CC [24]. The third study looked at the influence of Early Life Stress (ELS) on FA of the genu of the CC across the life span in healthy individuals. The results showed a lower FA in the youngest (8-12 years) and oldest (51-72 years) ELS age groups compared to non-exposed controls, suggesting that the effect is independent from the presence of psychopathology [44]. This was corroborated in the fourth study by Huang and Rao, in adolescents exposed to childhood maltreatment but without a history of psychiatric illness, who showed decreased FA values compared to controls in the left and right superior longitudinal fasciculi, right cingulum bundle, left inferior fronto-occipital fasciculus and splenium of the CC [21]. None of these studies investigated additional DTI parameters and only a region-of-interest (ROI) approach was used, which may have led important alterations in WM microstructure outside the ROI to go unobserved. Furthermore, study populations were heterogeneous for type of child adversity, which might have resulted in heterogeneous neuroimaging findings and differences in psychopathological sequelae.

The aim of our study was to investigate white matter integrity in a group of adolescents with psychopathology related to childhood sexual abuse (CSA) and matched healthy controls. We chose CSA as this is a prevalent form of child psychotrauma and a frequent cause of PTSD [15, 27]. This study is the first to focus on integrity of white matter tracts in a group of adolescents who had all experienced CSA. Based on previous neuroanatomical studies in children and youth as well as in adults, we hypothesized a reduced FA in the CC, the UF and the cingulum, although findings have not been unequivocal. We also aimed to investigate the possible relationship between FA and clinical symptoms in the patient group. Next, we also planned an exploratory whole brain analysis to detect aberrant FA values in areas outside our a priori defined ROIs.

\section{Methods}

\section{Participants}

We included $N=22$ adolescents with a history of CSA and related PTSD (further described as PTSD group) and $N=30$ healthy controls. The current cross-sectional study is part of the Emotional Pathways' Imaging Study in Clinical Adolescents (EPISCA), a longitudinal MRI study in which adolescents are followed over a 6-month period.

Inclusion criteria for the patient group were having experienced sexual abuse during their lifetime more than once by one or more perpetrators in- or outside the family and being referred to a mental health service. Most participants came from specialized psychotrauma centers and had experienced severe and frequent sexual abuse. Presence of PTSD was not an inclusion criterion, but clinical assessments (see below) showed that all patients but one were having PTSD related to the CSA. Exclusion criteria were: (1) primary DSM-IV diagnosis of ADHD, pervasive 
developmental disorders, Tourette's syndrome, obsessivecompulsive disorder, bipolar disorder, and psychotic disorders, (2) current use of psychotropic medication other than stable use of SSRI's, or amphetamine medication on the day of scanning, and (3) current substance abuse. The healthy control adolescents were recruited through local advertisement, with the following inclusion criteria: no clinical scores on validated mood and behavioral questionnaires, no history of traumatic experiences and no current psychotherapeutic intervention of any kind. All participants met the following inclusion criteria: aged between 12 and 21 , estimated full scale IQ (FIQ) $\geq 80$ as measured by Dutch versions of the Wechsler Intelligence Scales for Children (WISC-III) [59] or adults (WAIS) [58], being right-handed, normal or corrected-to-normal vision, sufficient understanding of the Dutch language, no history of neurological impairments and no contraindications for MRI testing (e.g. braces, metal implants or possible pregnancy). More extensive description of the clinical group can be found in an earlier report about the EPISCA project [57].

The medical ethics committee of the Leiden University Medical Centre approved the study. All anatomical scans were reviewed and cleared by a radiologist. Written informed consent was obtained from all adolescents and their parents. Participants received a financial compensation including travel expenses.

\section{Clinical assessments}

A standardized set of instruments was used to assess symptomatology in both groups of adolescents.

The Anxiety Disorders Interview Schedule Child and Parent Versions (ADIS-C/P) [46] are semi-structured interviews for the classification of DSM-IV anxiety and depressive disorders in children. The adolescents and at least one of their parents were interviewed. A minimal interference score of 4 , obtained by trained examiners based on the ADIS-C and ADIS-P, is necessary for classification. The ADIS is known to have good reliability and validity [47] with reported strong test-retest reliability statistics for the ADIS-C/P for combined diagnoses $(0.80-0.92)$ and individual diagnoses $(0.62-0.88)$.

As brain development is known to be influenced by sexual development, physical sexual development was measured with the self-report Puberty Development Scale (PDS) [38]. The PDS consists of five items that are measured on a five-point scale by the examiner: $1=$ pre-pubertal, $2=$ early pubertal, $3=$ mid-pubertal, $4=$ late pubertal, $5=$ post-pubertal. The PDS is considered a valuable instrument determining pubertal stage $[4,19]$.

The Trauma Symptom Checklist for Children (TSCC) [7] which measures trauma-related symptoms is a 54-item self-report for children and adolescents aged 8 through 18 , but is often used up to 21 years $[3,18]$. On a four-point scale (never to almost all of the time), the adolescent indicates how often a thought, a feeling or a behavior occurs. The items are grouped into six clinical scales. The clinical scales are Anxiety (Anx), Depression (Dep), Post-traumatic Stress (Pts), Sexual Concerns (Sc), Dissociation (Dis) and Anger (Ang). The TSCC total score is used as the main measure on post-traumatic symptomatology. Cronbach's alpha coefficients reported range from 0.77 to 0.89 for subscales and 0.84 for the total scale. The questionnaire has extensively been studied, which has confirmed its good psychometric qualities $[29,36]$.The internal consistency of the TSCC subscales varied between 0.85 and 0.94 , except for the Sexual Concerns subscale that measured 0.68.

Six subscales from the Wechsler Intelligence scales scores (picture completion, similarities, picture concepts, arithmetic, block design and comprehension) were converted into FIQ estimates.

\section{Data acquisition and preprocessing}

DTI data were collected using a Philips 3.0T Achieva MRI scanner (Philips Medical Systems, Best, The Netherlands) with an eight-channel SENSE (Sensitivity Encoding) head coil. A single-shot echo-planar imaging sequence was used with the following scan parameters: repetition time $=11,000 \mathrm{~ms}$, echo time $=56 \mathrm{~ms}$, flip angle $=90^{\circ}$, $b$-factor $=1000 \mathrm{~s} / \mathrm{mm}^{2}$, voxel dimensions $=2.3 \mathrm{~mm}$ isotropic, number of slices $=73$, and no slice gap. DTI data were acquired along 32 directions, together with a baseline image having no diffusion weighting $(b=0)$. Total scanning time was $\sim 7.5 \mathrm{~min}$. Collected DTI data were preprocessed and analyzed, using the Oxford Centre for Functional MRI of the Brain (FMRIB) software library (FSL; http:// fsl.fmrib.ox.ac.uk/fsl/fslwiki/) [49] version 5.0.2. First, DTI data were corrected for distortion and motion artifacts, induced by eddy currents or by simple head motions, using affine registration of each diffusion weighted image to the $b=0$ reference image. Next, non-brain tissue was removed, using the Brain Extraction Tool. Finally, to generate individual FA maps for each participant, the diffusion tensor model was fitted to each voxel, using FMRIB's Diffusion Toolbox. Total brain volume, normalized for subject head size, was estimated using SIENAX [51], part of FSL.

\section{Tract-based spatial statistics}

Tract-based spatial statistics (TBSS) [48] version 1.2 was used for voxelwise analysis of the preprocessed FA data. First, individual FA images were aligned to the FMRIB58_ FA standard-space image, using nonlinear registration. Next, the mean FA image was generated and thinned to create a mean FA skeleton, which represents the centers of 
all tracts common to the entire group. The mean FA skeleton was then thresholded at a FA value of $\geq 0.4$, to exclude peripheral tracts and minimize partial voluming. Finally, each participant's aligned FA images were projected onto the mean FA skeleton and the resulting data were fed into voxelwise permutation-based analysis.

\section{Region-of-interest TBSS}

To test for regional specific FA alterations, we implemented an ROI-based TBSS. A binary mask, encompassing the bilateral UF, the genu, splenium and body of the CC and the bilateral cingulum, was created as region of interest using the Johns Hopkins University (JHU) white matter atlas provided by FSL [34]. The uncinate fasciculus connects subcortical subregions of the limbic system, such as the hippocampus and the amygdala, with the medial prefrontal cortex. The corpus callosum is the largest white matter bundle in the brain and connects left and right cerebral hemispheres. It consists of three subregions, namely the splenium (posterior), the body (middle), and the genu (anterior). The cingulum bundle is situated superior to the corpus callosum, curving around the genu and splenium. It connects prefrontal and subcortical areas, with additional projections to the parietal lobe.

The mask was then applied to the mean FA skeleton, to include only voxels comprised in the mean FA skeleton. This confines the statistical analysis exclusively to voxels from the center of the tract, thereby minimizing anatomic inter-subject variability, registration errors, and partial voluming. The resulting study-specific ROI mask was used for voxelwise permutation-based ROI analysis.

\section{Statistical analysis of demographic and clinical data}

We used analysis of variance (ANOVA) to compare the two groups on age, IQ and TSCC total score. Because not all TSCC subscales showed normal distribution we used nonparametric analysis (Mann-Whitney) for the comparison of the TSCC subscales between the two groups.

\section{MRI analysis}

Using FSL's Randomize tool, permutation-based inferences with Threshold-Free Cluster Enhancement (TFCE) were carried out for voxelwise analysis of FA data [50]. 5000 random permutations were generated to build up the null distribution of the cluster size statistic, while testing the following contrasts: (1) controls > PTSD, (2) controls < PTSD. PDS score, total brain volume, gender and FIQ (demeaned across groups) were included in the analysis as nuisance regressors to correct for between groups variances. The resulting statistical maps were corrected for multiple comparisons across space $(p<0.05)$ and the JHU White Matter and Juelich Histological atlases were used to label clusters with significant FA alterations. This step was first carried out using the ROI mask to test our specific hypotheses. Next, we ran this step a second time using a whole brain mask for our exploratory analysis.

\section{Post hoc analyses}

The association between FA and symptom severity in the PTSD group was examined using a voxelwise correlation approach. A mask was created of the voxels that were found to differ significantly on FA based on the betweengroup ROI analysis.

The TSCC total and subscale scores of the PTSD group were fed into FSL's Randomize tool along with the mask, using permutation-based inferences with TFCE.

Last, we examined how the between-group differences in FA values related to the other DTI measures. Therefore, information on each individuals' AD (the 1st eigenvalue), $\mathrm{RD}$ (the average of the 2nd and 3rd eigenvalues), and MD was fed into FSL's Randomize tool along with the mask based on our ROI analysis.

\section{Results}

From the original total of 22 PTSD and 30 control adolescents, three controls were excluded because of image artifacts in T1-weighted anatomical scans. Further, two adolescents with PTSD were excluded because of image artifacts in the DTI dataset, resulting in a final sample of 20 adolescents with PTSD. From the remaining 27 controls, 20 subjects were group-wise matched on age and gender with the PTSD adolescents. Eventually, 40 participants (20 PTSD and 20 controls) were included. Of the 20 PTSD participants, 19 fulfilled all PTSD criteria on the ADIS, while one had sufficient PTSD symptoms, but with limited interference. Since earlier research showed that persons with subthreshold PTSD in many aspects resemble PTSD patients, we decided to include this patient [10].

The majority of participants was female $(88 \%$, see Table 1). Of the participants with PTSD, 15 had comorbid anxiety disorders, most often more than one. Eight had a comorbid depressive disorder and one an oppositional defiant disorder (ODD). All controls and 16 adolescents with PTSD were drug and treatment naïve. Two adolescents with PTSD were on stable SSRI treatment and two used amphetamines (not on the day of scanning).

The PTSD group had a significantly lower FIQ than controls $[F(1,38)=8,14, p<0.01]$ and more subjects in the post-pubertal phase (50 versus $25 \%)$. As expected, there was a significant main effect of group on the TSCC scale scores [with and without controlling for age and FIQ; $F(7,28)=6,48, p<0.01]$. The PTSD group had 
Table 1 Demographic and clinical characteristics of participants

\begin{tabular}{|c|c|c|c|c|c|}
\hline & \multicolumn{2}{|c|}{$\operatorname{PTSD}(N=20)$} & \multicolumn{2}{|c|}{$\operatorname{CNTR}(N=20)$} & \multirow[t]{2}{*}{$p$} \\
\hline & Mean & SD & Mean & SD & \\
\hline Gender (f : m) & $17: 3$ & & $18: 2$ & & \\
\hline Age (in months) & 198 & 24 & 185 & 19 & 0.06 \\
\hline FIQ & 99 & 9 & 107 & 9 & $<0.01$ \\
\hline \multicolumn{6}{|l|}{$P D S^{\mathrm{a}}$} \\
\hline Pre/mid-pubertal & 1 & & 4 & & \\
\hline Late pubertal & 6 & & 9 & & \\
\hline Post-pubertal & 10 & & 5 & & \\
\hline \multicolumn{6}{|l|}{$T S C C^{\mathrm{b}}$} \\
\hline Anxiety & 9.3 & & 6.0 & & 3.3 \\
\hline Depression & 9.9 & & 4.9 & & 2.6 \\
\hline Anger & 6.2 & & 3.5 & & 2.0 \\
\hline Post-traumatic stress & 11.8 & & 7.2 & & 2.3 \\
\hline Dissociation & 8.6 & & 5.6 & & 2.7 \\
\hline Sexual concerns & 4.7 & & 3.3 & & 1.3 \\
\hline
\end{tabular}

Because less than $20 \%$ of the data in TSCC were missing, expectation maximization as regression method was used to calculate the scale scores

a Missing data: 2 in control group, 3 in PTSD group

b Three PTSD participants did not complete the questionnaire

significantly higher scores on all TSCC scales (all with $p<0.01)$.

\section{TBSS Analyses}

ROI-based TBSS analysis showed that, in comparison with controls, the PTSD group had lower FA values in the genu, midbody and splenium of the CC $(p<0.05$, TFCE corrected) (Fig. 1). No FA differences were observed in the bilateral UF and cingulum. The exploratory whole brain TBSS revealed no significant lower FA values. When the threshold was lowered we found lower FA values in the body of the CC in the left hemisphere, adjacent to the splenium ( $p<0.075$, TFCE corrected; Fig. 2). No white matter tracts with lower FA values were found for controls versus PTSD.

Using a voxelwise correlation approach, we examined the association between the observed smaller FA values from the ROI analysis, and the TSCC total and subscale scores in the patients. We found a significant negative correlation between scores on the anger subscale of the TSCC and FA values in the left body of the CC $(p<0.05$; uncorrected, Fig. 3.)

Post hoc analyses of the AD, RD and MD in the voxels that showed FA differences between groups revealed a significant increase ( $p<0.05$, TFCE corrected) of RD and MD in the PTSD group compared to controls. No significant differences were found between groups in AD. Omitting the one CSA participant who met all PTSD criteria except for interference did not change our findings. Excluding the two participants that were using medication from the analyses did not change the results either.

\section{Discussion}

We examined white matter integrity in a sample of adolescents with CSA-related PTSD, using an ROI and an additional exploratory whole brain approach. We hypothesized reduced FA in a number of relevant white matter tracts: the CC, UF and cingulum. Compared to the control group, our adolescent PTSD group only showed decreased FA in areas of the CC, with additional DTI parameters suggesting demyelinization and dysmyelinization in these areas. We also found a significant correlation (uncorrected) between FA in the CC and Anger scores on the TSCC in the adolescents with CSA-related PTSD.

This study is the first to report on white matter integrity in a group of adolescents with CSA-related PTSD. The results of our study are in line with the findings of the recent DTI study by Jackowski and colleagues, who examined the CC in a group of children with PTSD following various forms of intrafamilial maltreatment, and also found reduced FA in several subregions of the CC [24]. Our findings are in line with recent reviews indicating that the most consisting finding in youth with psychotrauma is structural abnormalities of the $\mathrm{CC}$, in contrast to the reduction of hippocampal volume typically reported in adults with PTSD $[5,40]$.

The CC is known to change throughout life, but most dramatically during childhood and adolescence [2, 30]. These developmental changes in the $\mathrm{CC}$ are the consequence of varying degrees of axonal myelinization, redirection, and pruning, reflecting a permanent adjustment and fine-tuning of fibers connecting homologous cortical areas. The general trend during adolescence is toward increasing FA and decreasing MD [43]. This CC maturation parallels puberty development suggesting gonadal hormonal influences [2]. For this reason, we included PDS scores as regressor. However, we must acknowledge that a simple linear regression of pubertal stage and total brain volume may still not sufficiently account for the results as they are known not to be linear across adolescence.

Early traumatization is likely to have a major influence on the integrity of the $\mathrm{CC}$, as the processes of myelinization and selective pruning are typically influenced by stress hormones [52, 54]. Of importance, the smaller FA values we found in the CC of the PTSD group were due to increases in $\mathrm{RD}$ and $\mathrm{MD}$, known to reflect demyelinization (less development of the myelin sheet) and dysmyelinization 


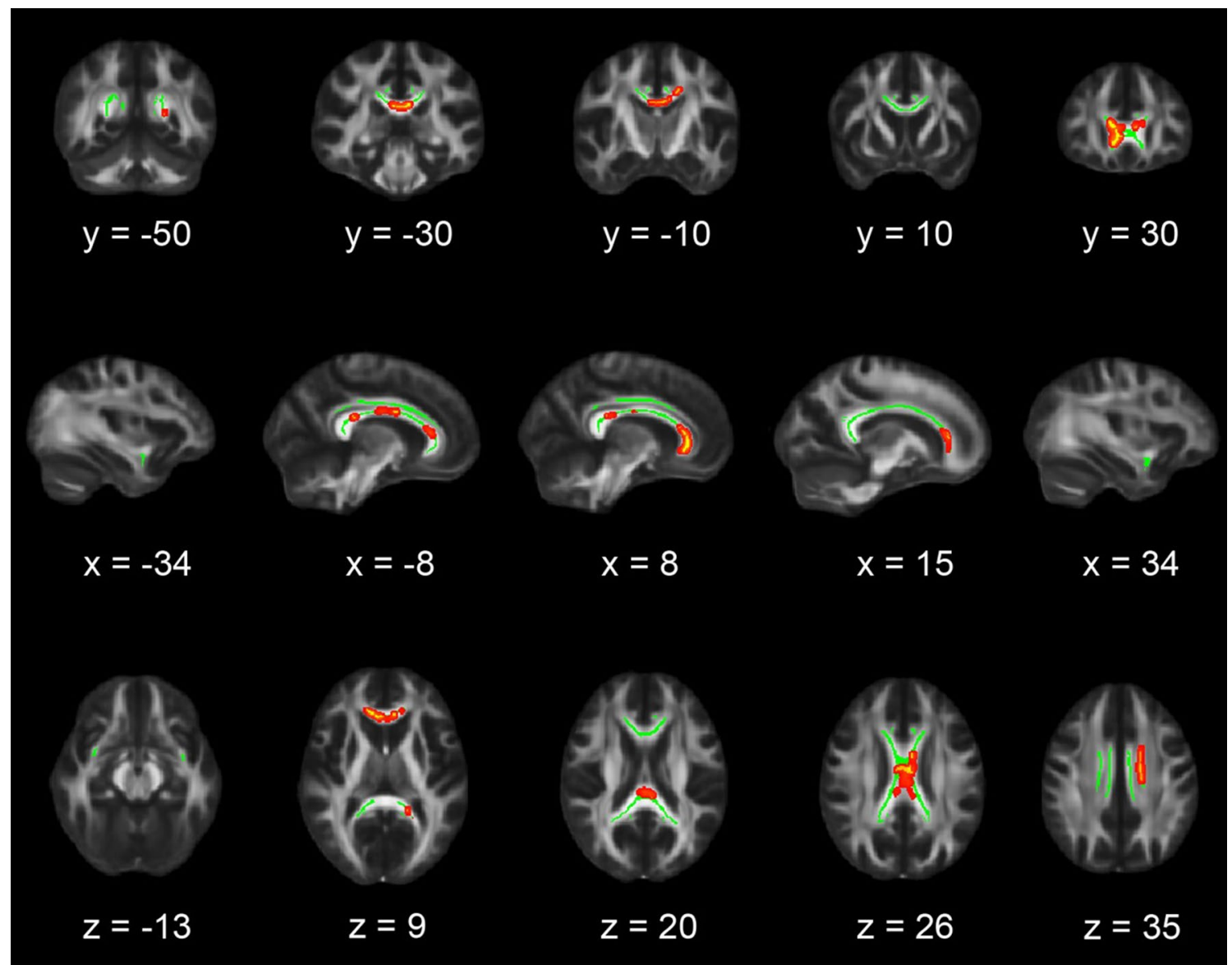

Fig. 1 Region-of-interest analysis results. Coronal, sagittal and transversal axial sections of the white matter skeleton (green) superimposed on the FMRIB58_FA_1 mm standard brain (gray). Depicted in yellow are the regions in which FA values are significantly smaller in patients with PTSD compared to matched healthy controls. For better visibility, the results are thickened using the "tbss-fill" command (red). All TBSS results are corrected for multiple comparisons $(p<0.05$, TFCE corrected), and the axial images are in radiological convention (the right side of the image corresponds with the left hemisphere of the brain and vice versa)

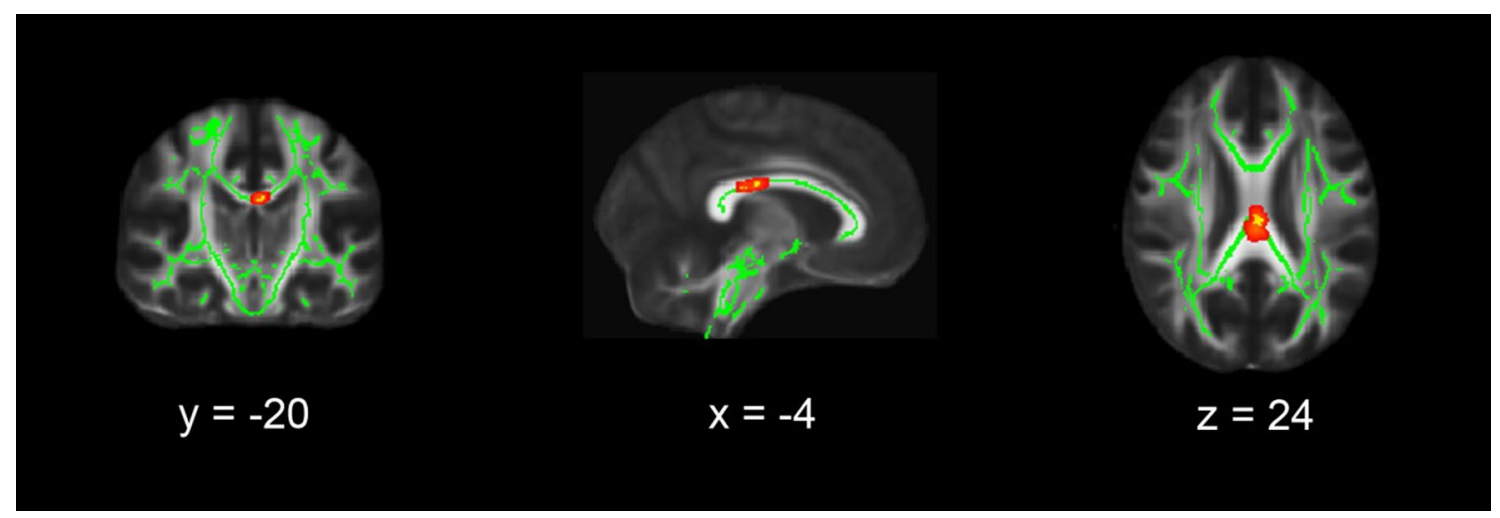

Fig. 2 Whole brain TBSS results. Coronal, sagittal and transversal axial sections of the white matter skeleton (green) superimposed on the FMRIB58_FA_1 mm standard brain (gray). Depicted in yellow are the regions in which FA values are significantly smaller in patients with PTSD compared to matched healthy controls. For bet- ter visibility, the results are thickened using the "tbss-fill" command (red) All TBSS results are corrected for multiple comparisons $(p<0.075$, TFCE corrected), and the axial images are in radiological convention (the right side of the image corresponds with the left hemisphere of the brain and vice versa) 


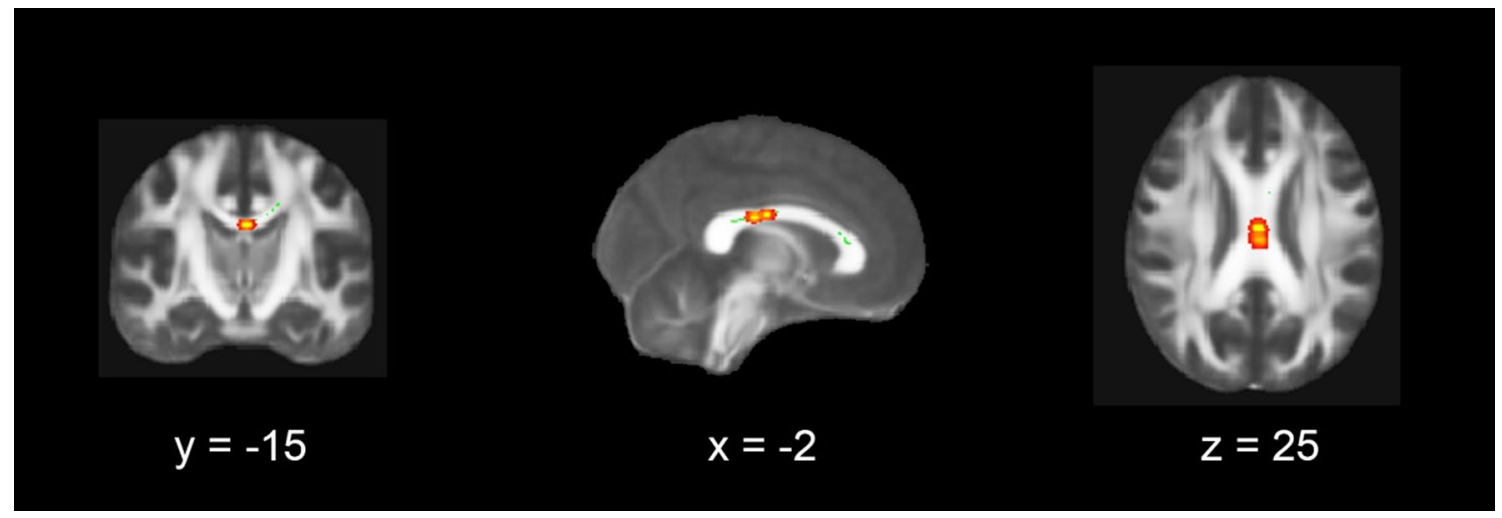

Fig. 3 Voxel-wise correlation between TSCC anger subscale scores and FA values in adolescents with PTSD. Coronal, sagittal and transversal axial sections of the white matter skeleton (green) superimposed on the FMRIB58_FA_1 mm standard brain (gray). FA values in the left CC correlated negatively with TSCC anger subscale scores

(aberrant development of the myelin sheet), linking the abnormalities of the $\mathrm{CC}$ integrity to the possible influence of stress hormones. Supporting this possible association, a recent study found that in rhesus monkeys exposed to early maternal abuse, cortisol levels at the time of abuse correlated with abnormalities in white matter connectivity in the CC, brain stem and other brain areas in adolescence [20]. Our results are in line with the study of Teicher et al. who, comparing abuse and neglect, found that sexual abuse was the strongest factor influencing CC size in girls [53].

Recent topographic research on the CC is beginning to map the different regions of the $\mathrm{CC}$ and their connections. Apart from frontal connections, the body of the $\mathrm{CC}$ also has connections with subcortical nuclei [21]. Changes in the midbody of the CC in children and adolescents who experienced psychological trauma could be related to disturbances in connectivity with limbic subcortical nuclei, resulting from or underlying the disturbances in emotion regulation.

We found a negative association in the adolescent PTSD group between FA in the CC and the TSCC Anger subscale. This in contrast to the result of the small DTI study in socioemotional deprived children with PTSD [14] in which correlations of FA measures in the $\mathrm{CC}$ were found with total anxiety scores, panic scores and separation anxiety scores. This may be due to methodological differences as well as the populations studied. A preclinical study in male nonhuman primates examined the effects of early life stress on hippocampal volume and $\mathrm{CC}$ development and found a significant inverse relationship between CC mid-sagittal area in adult monkeys and the response toward an intruder which typically consists of a mixture of aggressive and anxious behavior [23]. In a recent DTI study in male adolescents with conduct disorder, Zhang et al. report increased structural connectivity in the genu and body of the CC [61]. $(p<0.05)$ in the adolescents with PTSD (yellow). For better visibility, the results are thickened using the "tbss-fill" command (red). The axial images are in radiological convention (the right side of the image corresponds with the left hemisphere of the brain and vice versa)

Impulsivity correlated positive with WM integrity, which is the opposite pattern of what we found in our study. This suggests that different pathophysiological mechanisms are involved, which is in accordance with the putative mechanisms described in the literature. For instance, Raine et al. hypothesize that structural abnormalities in the $\mathrm{CC}$ are a consequence of an early arrest of the normal neurodevelopmental process of axonal pruning, while the abnormalities in myelinization following CSA may be more linked to detrimental stress hormone influences [39].

We believe the relatively homogeneous sample and the state-of-the-art DTI approaches are strengths of our study, although several potential limitations should be taken into account. While we know that gender influences brain development and the reaction to psychological trauma, we included gender as a regressor, but could not further explore this issue because our participants are mainly girls. Full scale IQ measures differed significantly between the PTSD group and controls. Several studies report a negative effect of ELS on cognitive function [13, 37]. In this respect intellectual ability in the PTSD group and the control group might originally have been more equal. The New Zealand longitudinal birth cohort study [28] instead points to IQ as a risk factor for the development of PTSD. In the discussion about lower IQ being a consequence or a predictor of PTSD, it is suggested that trauma severity overrules IQ as a predictor [33] which could be the case in our study where all included adolescents fulfilled symptom criteria for PTSD diagnosis although this was not an inclusion criterion, but here too results are inconclusive [6]. NavasSanchez et al. [35] found a positive correlation between IQ and FA in the $\mathrm{CC}$ in math gifted adolescents compared to controls matched for age and academic level. Other studies report about correlations, mostly positive, of cognitive 
function with FA in several WM tracts [17, 42]. To decrease the potential influence of IQ on the white matter integrity differences we included IQ as a covariate in our analyses, but clearly further research is needed to unravel the exact relationship between childhood adversity, IQ and WM integrity in adolescence.

The normal increase of FA in the CC during adolescence is related to pubertal development. Therefore, the PDS score is chosen as nuisance regressor instead of age. The PTSD group was older and more advanced in pubertal stage. Because of the expected increase, the decrease in FA found in the PTSD group cannot be a consequence of normal (pubertal) development.

Two adolescents with PTSD were on stable SSRI treatment. Omitting these two participants from our analyses did not have any effect on the results. To our knowledge no influence of SSRIs on the CC is reported [9].

Patients were selected based on the presence of CSA and all except one, who fulfilled PTSD criteria except interference, showed CSA-related PTSD. Hence, we cannot differentiate whether the neuroimaging results were a consequence of exposure to trauma or the (development of) PTSD pathology or reflect an underlying vulnerability. Previous research in twins discordant for combat exposure suggests that anatomical abnormalities may indeed represent pre-existing vulnerability factors [16]. The ideal crosssectional design to disentangle the effects of exposure, psychopathology and resilience would have incorporated a CSA group with psychopathology, a CSA group without psychopathology and a non-exposed, healthy control group [55]. Furthermore, we could not assess the influence of timing and duration of the CSA in our study, which is thought to be highly relevant in children and youth.

About one-third of the subjects also reported physical abuse, but as we did not assess experiences of other forms of psychotrauma we could have missed other prevalent traumatic experiences, like emotional maltreatment, which might be associated with the presence of DTI abnormalities in our sample.

In conclusion, our DTI findings in this sample of adolescents with CSA-related PTSD point at the involvement of the $\mathrm{CC}$ in brain alterations associated with juvenile sexual psychotrauma, and together with recent animal data can be taken to point at the influence of stress hormone levels on CC integrity. Clearly, longitudinal studies till mid-adulthood are needed to further elucidate the role of altered CC white matter integrity in the biopsychological consequences of early traumatization and to examine its malleability.

Acknowledgments Adolescents and their parents involved in the EPISCA study are gratefully acknowledged, as well as the participating centers: the Department of Child and Adolescent Psychiatry and the Psychotrauma Center of GGZ Rivierduinen, the Kind en Jeugd Traumacentrum (KJTC) in Haarlem, the LUMC Departments of Psychiatry and Radiology, and the Leiden Institute for Brain and Cognition. The EPISCA project is funded by a grant from the LUMC. M. J. van Hoof was supported by WOP GGZ Rivierduinen, which also made assistance in patient recruitment, data collection and data management by C. I. Gelderblom possible. Two researchers in this study were also supported through The Netherlands Organization for Scientific Research: S. A. Rombouts NWO Vici project (grant number 016130677) and S. J. A van der Werff: National Initiative Brain and Cognition projects (Grant number 056-25-010). The authors also gratefully acknowledge the financial support given by the participating centers.

\section{Compliance with ethical standards}

\section{Conflict of interest None.}

Open Access This article is distributed under the terms of the Creative Commons Attribution 4.0 International License (http://creativecommons.org/licenses/by/4.0/), which permits unrestricted use, distribution, and reproduction in any medium, provided you give appropriate credit to the original author(s) and the source, provide a link to the Creative Commons license, and indicate if changes were made.

\section{References}

1. Anda RF, Felitti VJ, Bremner JD, Walker JD, Whitfield C, Perry BD, Dube SR, Giles WH (2006) The enduring effects of abuse and related adverse experiences in childhood. A convergence of evidence from neurobiology and epidemiology. Eur Arch Psychiatry Clin Neurosci 256:174-186

2. Asato MR, Terwilliger R, Woo J, Luna B (2010) White matter development in adolescence: a DTI study. Cereb Cortex 20:2122-2131

3. Barakat LP, Kazak AE, Meadows AT, Casey R, Meeske K, Stuber ML (1997) Families surviving childhood cancer: a comparison of posttraumatic stress symptoms with families of healthy children. J Pediatr Psychol 22:843-859

4. Bond L, Clements J, Bertalli N, Evans-Whipp T, McMorris BJ, Patton GC, Toumbourou JW, Catalano RF (2006) A comparison of self-reported puberty using the Pubertal Development Scale and the Sexual Maturation Scale in a school-based epidemiologic survey. J Adolesc 29:709-720

5. Bremner JD (2007) Neuroimaging in posttraumatic stress disorder and other stress-related disorders. Neuroimaging Clin N Am 17:523-38, ix

6. Breslau N, Chen Q, Luo Z (2013) The role of intelligence in posttraumatic stress disorder: does it vary by trauma severity? PLoS ONE 8:e65391

7. Briere J (1996) Trauma Symptoms Checklist for Children (TSCC), Professional Manual. Psychological Assessment Resources, Odessa

8. Casey BJ, Jones RM, Hare TA (2008) The adolescent brain. Ann N Y Acad Sci 1124:111-126

9. Chen $\mathrm{CH}$, Ridler K, Suckling J, Williams S, Fu CH, Merlo-Pich E, Bullmore E (2007) Brain imaging correlates of depressive symptom severity and predictors of symptom improvement after antidepressant treatment. Biol Psychiatry 62:407-414

10. Cukor J, Wyka K, Jayasinghe N, Difede J (2010) The nature and course of subthreshold PTSD. J Anxiety Disord 24:918-923

11. Daniels JK, Lamke JP, Gaebler M, Walter H, Scheel M (2013) White matter integrity and its relationship to ptsd and childhood trauma-a systematic review and meta-analysis. Depress Anxiety 30:207-216 
12. De Bellis MD, Spratt EG, Hooper SR (2011) Neurodevelopmental biology associated with childhood sexual abuse. J Child Sex Abus 20:548-587

13. De Bellis MD, Woolley DP, Hooper SR (2013) Neuropsychological findings in pediatric maltreatment: relationship of PTSD, dissociative symptoms, and abuse/neglect indices to neurocognitive outcomes. Child Maltreat 18:171-183

14. Eluvathingal TJ, Chugani HT, Behen ME, Juhasz C, Muzik O, Maqbool M, Chugani DC, Makki M (2006) Abnormal brain connectivity in children after early severe socioemotional deprivation: a diffusion tensor imaging study. Pediatrics 117:2093-2100

15. Gibb BE, Chelminski I, Zimmerman M (2007) Childhood emotional, physical, and sexual abuse, and diagnoses of depressive and anxiety disorders in adult psychiatric outpatients. Depress Anxiety $24: 256-263$

16. Gilbertson MW, Shenton ME, Ciszewski A, Kasai K, Lasko NB, Orr SP, Pitman RK (2002) Smaller hippocampal volume predicts pathologic vulnerability to psychological trauma. Nat Neurosci 5:1242-1247

17. Glascher J, Rudrauf D, Colom R, Paul LK, Tranel D, Damasio H, Adolphs R (2010) Distributed neural system for general intelligence revealed by lesion mapping. Proc Natl Acad Sci USA 107:4705-4709

18. Gustafsson PE, Nilsson D, Svedin CG (2009) Polytraumatization and psychological symptoms in children and adolescents. Eur Child Adolesc Psychiatry 18:274-283

19. Herting MM, Maxwell EC, Irvine C, Nagel BJ (2012) The impact of sex, puberty, and hormones on white matter microstructure in adolescents. Cereb Cortex 22:1979-1992

20. Howell BR, McCormack KM, Grand AP, Sawyer NT, Zhang X, Maestripieri D, Hu X, Sanchez MM (2013) Brain white matter microstructure alterations in adolescent rhesus monkeys exposed to early life stress: associations with high cortisol during infancy. Biol Mood Anxiety Disord 3:21

21. Huang H, Zhang J, Jiang H, Wakana S, Poetscher L, Miller MI, van Zijl PC, Hillis AE, Wytik R, Mori S (2005) DTI tractography based parcellation of white matter: application to the mid-sagittal morphology of corpus callosum. Neuroimage 26:195-205

22. Hulvershorn LA, Cullen K, Anand A (2011) Toward dysfunctional connectivity: a review of neuroimaging findings in pediatric major depressive disorder. Brain Imaging Behav 5:307-328

23. Jackowski A, Perera TD, Abdallah CG, Garrido G, Tang CY, Martinez J, Mathew SJ, Gorman JM, Rosenblum LA, Smith EL, Dwork AJ, Shungu DC, Kaffman A, Gelernter J, Coplan JD, Kaufman J (2011) Early-life stress, corpus callosum development, hippocampal volumetrics, and anxious behavior in male nonhuman primates. Psychiatry Res 192:37-44

24. Jackowski AP, Douglas-Palumberi H, Jackowski M, Win L, Schultz RT, Staib LW, Krystal JH, Kaufman J (2008) Corpus callosum in maltreated children with posttraumatic stress disorder: a diffusion tensor imaging study. Psychiatry Res 162:256-261

25. Kaufman J, Plotsky PM, Nemeroff CB, Charney DS (2000) Effects of early adverse experiences on brain structure and function: clinical implications. Biol Psychiatry 48:778-790

26. Kessler RC, McLaughlin KA, Green JG, Gruber MJ, Sampson NA, Zaslavsky AM, Aguilar-Gaxiola S, Alhamzawi AO, Alonso J, Angermeyer M, Benjet C, Bromet E, Chatterji S, de Girolamo G, Demyttenaere K, Fayyad J, Florescu S, Gal G, Gureje O, Haro JM, Hu CY, Karam EG, Kawakami N, Lee S, Lepine JP, Ormel J, Posada-Villa J, Sagar R, Tsang A, Ustun TB, Vassilev S, Viana MC, Williams DR (2010) Childhood adversities and adult psychopathology in the WHO World Mental Health Surveys. Br J Psychiatry 197:378-385

27. Kessler RC, Sonnega A, Bromet E, Hughes M, Nelson CB (1995) Posttraumatic stress disorder in the National Comorbidity Survey. Arch Gen Psychiatry 52:1048-1060
28. Koenen KC, Moffitt TE, Poulton R, Martin J, Caspi A (2007) Early childhood factors associated with the development of posttraumatic stress disorder: results from a longitudinal birth cohort. Psychol Med 37:181-192

29. Lanktree CB, Gilbert AM, Briere J, Taylor N, Chen K, Maida CA, Saltzman WR (2008) Multi-informant assessment of maltreated children: convergent and discriminant validity of the TSCC and TSCYC. Child Abuse Negl 32:621-625

30. Luders E, Thompson PM, Toga AW (2010) The development of the corpus callosum in the healthy human brain. J Neurosci 30:10985-10990

31. MacMillan HL, Fleming JE, Streiner DL, Lin E, Boyle MH, Jamieson E, Duku EK, Walsh CA, Wong MY, Beardslee WR (2001) Childhood abuse and lifetime psychopathology in a community sample. Am J Psychiatry 158:1878-1883

32. McCrory E, De Brito SA, Viding E (2010) Research review: the neurobiology and genetics of maltreatment and adversity. J Child Psychol Psychiatry 51:1079-1095

33. McNally RJ, Shin LM (1995) Association of intelligence with severity of posttraumatic stress disorder symptoms in Vietnam Combat veterans. Am J Psychiatry 152:936-938

34. Mori SWS, Nagae-Poetscher LM, van Zijl PCM (2005) MRI atlas of human white matter. Elsevier, Amsterdam

35. Navas-Sanchez FJ, Aleman-Gomez Y, Sanchez-Gonzalez J, Guzman-De-Villoria JA, Franco C, Robles O, Arango C, Desco M (2014) White matter microstructure correlates of mathematical giftedness and intelligence quotient. Hum Brain Mapp 35:2619-2631

36. Nilsson D, Wadsby M, Svedin CG (2008) The psychometric properties of the Trauma Symptom Checklist For Children (TSCC) in a sample of Swedish children. Child Abuse Negl 32:627-636

37. Pechtel P, Pizzagalli DA (2011) Effects of early life stress on cognitive and affective function: an integrated review of human literature. Psychopharmacology 214:55-70

38. Petersen AC (1988) Adolescent development. Annu Rev Psychol 39:583-607

39. Raine A, Lencz T, Taylor K, Hellige JB, Bihrle S, Lacasse L, Lee M, Ishikawa S, Colletti P (2003) Corpus callosum abnormalities in psychopathic antisocial individuals. Arch Gen Psychiatry 60:1134-1142

40. Rinne-Albers MA, van der Wee NJ, Lamers-Winkelman F, Vermeiren RR (2013) Neuroimaging in children, adolescents and young adults with psychological trauma. Eur Child Adolesc Psychiatry 22(12):745-755

41. Sanchez MM, Ladd CO, Plotsky PM (2001) Early adverse experience as a developmental risk factor for later psychopathology: evidence from rodent and primate models. Dev Psychopathol 13:419-449

42. Schmithorst VJ, Wilke M, Dardzinski BJ, Holland SK (2005) Cognitive functions correlate with white matter architecture in a normal pediatric population: a diffusion tensor MRI study. Hum Brain Mapp 26:139-147

43. Schmithorst VJ, Yuan W (2010) White matter development during adolescence as shown by diffusion MRI. Brain Cogn 72:16-25

44. Seckfort DL, Paul R, Grieve SM, Vandenberg B, Bryant RA, Williams LMCCR, Cohen RA, Bruce S, Gordon E (2008) Early life stress on brain structure and function across the lifespan: a preliminary study. Brain Imaging Behav 2:49-58

45. Shonkoff JP (2011) Protecting brains, not simply stimulating minds. Science 333:982-983

46. Silverman WK, Ollendick TH (2005) Evidence-based assessment of anxiety and its disorders in children and adolescents. $\mathbf{J}$ Clin Child Adolesc Psychol 34:380-411 
47. Silverman WK, Saavedra LM, Pina AA (2001) Test-retest reliability of anxiety symptoms and diagnoses with the Anxiety Disorders Interview Schedule for DSM-IV: child and parent versions. J Am Acad Child Adolesc Psychiatry 40:937-944

48. Smith SM, Jenkinson M, Johansen-Berg H, Rueckert D, Nichols TE, Mackay CE, Watkins KE, Ciccarelli O, Cader MZ, Matthews PM, Behrens TE (2006) Tract-based spatial statistics: voxelwise analysis of multi-subject diffusion data. Neuroimage 31:1487-1505

49. Smith SM, Jenkinson M, Woolrich MW, Beckmann CF, Behrens TE, Johansen-Berg H, Bannister PR, De LM, Drobnjak I, Flitney DE, Niazy RK, Saunders J, Vickers J, Zhang Y, De SN, Brady JM, Matthews PM (2004) Advances in functional and structural MR image analysis and implementation as FSL. Neuroimage 23(Suppl 1):S208-S219

50. Smith SM, Nichols TE (2009) Threshold-free cluster enhancement: addressing problems of smoothing, threshold dependence and localisation in cluster inference. Neuroimage 44:83-98

51. Smith SM, Zhang Y, Jenkinson M, Chen J, Matthews PM, Federico A, De SN (2002) Accurate, robust, and automated longitudinal and cross-sectional brain change analysis. Neuroimage $17: 479-489$

52. Teicher MH, Andersen SL, Polcari A, Anderson CM, Navalta CP (2002) Developmental neurobiology of childhood stress and trauma. Psychiatr Clin North Am 25:397-viii

53. Teicher MH, Dumont NL, Ito Y, Vaituzis C, Giedd JN, Andersen SL (2004) Childhood neglect is associated with reduced corpus callosum area. Biol Psychiatry 56:80-85

54. van der Werff SJ, Andela CD, Nienke PJ, Meijer OC, van Buchem MA, Rombouts SA, van der Mast RC, Biermasz NR,
Pereira AM, van der Wee NJ (2014) Widespread reductions of white matter integrity in patients with long-term remission of Cushing's disease. Neuroimage Clin 4:659-667

55. van der Werff SJ, Pannekoek JN, Veer IM, van Tol MJ, Aleman A, Veltman DJ, Zitman FG, Rombouts SA, Elzinga BM, van der Wee NJ (2012) Resting-state functional connectivity in adults with childhood emotional maltreatment. Psychol Med 43(9):1825-1836

56. van Harmelen AL, van Tol MJ, van der Wee NJ, Veltman DJ, Aleman A, Spinhoven P, van Buchem MA, Zitman FG, Penninx BW, Elzinga BM (2010) Reduced medial prefrontal cortex volume in adults reporting childhood emotional maltreatment. Biol Psychiatry 68:832-838

57. van Hoof MJ, van Lang ND, Speekenbrink S, van IJzendoorn MH, Vermeiren RR (2015) Adult Attachment Interview differentiates adolescents with Childhood Sexual Abuse from those with clinical depression and non-clinical controls. Attach Hum Dev 17:354-375

58. Wechsler D (1991) Wechsler Adult Intelligence Scale. The Psychological Corporation, San Antonio

59. Wechsler D (1997) Wechsler Intelligence Scale for Children. The Psychological Corporation, San Antonio

60. Woon FL, Hedges DW (2008) Hippocampal and amygdala volumes in children and adults with childhood maltreatment-related posttraumatic stress disorder: a meta-analysis. Hippocampus 18:729-736

61. Zhang J, Zhu X, Wang X, Gao J, Shi H, Huang B, Situ W, Yi J, Zhu X, Yao S (2014) Increased structural connectivity in corpus callosum in adolescent males with conduct disorder. J Am Acad Child Adolesc Psychiatry 53:466-475 